\title{
SEGURIDAD HUMANA Y CATÁSTROFES: LA LABOR DE LAS NACIONES UNIDAS EN MATERIA DE GESTIÓN Y REGULACIÓN DE LAS CATÁSTROFES
}

\author{
Andrés Bautista-Hernáez ${ }^{1}$
}

\section{Resumen}

Las catástrofes suponen un fenómeno que golpea a los miembros de la Sociedad Internacional en su conjunto. Es por ello que resulta necesario establecer mecanismos de gestión de catástrofes coordinados y efectivos no sólo en los ámbitos local, regional o nacional, sino también y más importante a nivel internacional. A pesar de su importancia, las normas internacionales en materia de catástrofes siguen siendo muy deficientes. Ello es debido a la falta de un cuerpo jurídico bien establecido y de un conjunto de reglas codificadas que permitan una regulación amplia y general. El papel que deba y juega la Organización de Naciones Unidas en materia de catástrofes resulta fundamental en este contexto. En este trabajo se analizará la labor de esta Organización en su doble aspecto el institucional y el normativo

\section{Abstract}

Members of International society are hit by consequences of disasters causing great disruption in their normal functioning. This is one of the reasons why it is necessary to set up mechanisms of disaster management in coordination within the local, regional, national and international level. In addition, there is a lack of a clear international set of rules governing disasters in a general way. In this context, it is essential the role played by United Nations in regards to the management and regulation of disasters. The main object of this paper is to analyse the current functions of United Nations on disasters. We will focus on the institutional and normative development on this duty in the four phases of disaster (prevention, preparedness, response and reconstruction). We shall try to

\footnotetext{
${ }^{1}$ Andrés Bautista-Hernáez es Profesor Sustituto Interino del área de Derecho Internacional Público y Relaciones Internacionales de la Universidad de Málaga (España) e investigador del Centre de Droit International de Nanterre de la Université Paris Nanterre (Francia); e-mail: abautista@uma.es.
} 
answer how UN manages disaster related issues and if it is done effectively, according to its global role on the international sphere.

\section{Palabras clave}

Catástrofes, Comité Permanente entre Organismos, Derecho Internacional de Catástrofes, Naciones Unidas, Oficina de Coordinación de NN.UU. de Asuntos Humanitarios (UNOCHA), Prevención, Preparación, Respuesta

\section{Keywords}

Disasters, Inter-Agengy Standing Committee (IASC), International Disaster Law, United Nations, United Nations Office for the Coordination of Humanitarian Affairs (UNOCHA), Prevention, Preparedness, Response

\section{Introducción: Relaciones entre CATÁStrofes Y Derecho INTERNACIONAL}

Las catástrofes suponen un fenómeno que golpea a los miembros de la Sociedad Internacional en su conjunto, sin importar la zona geográfica o el grado de desarrollo del Estado en cuestión. Además, se añade el elemento transfronterizo de las mismas afectando a varios Estados colindantes. Un claro ejemplo de esto lo encontramos en los recientes incendios forestales acaecidos en Portugal en Junio de 2017 y en Galicia (España) en agosto del mismo añoº

La regulación de las catástrofes ha estado ligada desde sus orígenes a la noción de humanidad. Ello se reflejó en la respuesta que se ofrecía frente a estos sucesos por parte

\footnotetext{
${ }^{2}$ Sobre la cooperación transfronteriza en materia de catástrofes, puede consultarse BAUTISTA-HERNÁEZ, A., «Cooperación transfronteriza y cooperación internacional frente a catástrofes en las relaciones LusoEspañolas. Consideraciones en torno al Protocolo de Évora de 1992», en FERNÁNDEZ SÁNCHEZ, P.-A. y AZEREDO LOPES, J.-A., Seguridad medioambiental y cooperación transfronteriza. IV Encuentro LusoEspañol de Profesores de Derecho Internacional Público y Relaciones Internacionales, Atelier, Barcelona, 2015, pp. 49-82.
} 
de la sociedad ${ }^{3}$ y a una naciente concienciación respecto de los deberes morales de las naciones. Precisamente, como plasmó el jurista suizo E. de VATTEL en su célebre obra en 1758 , indicando que

«[... Toute nation doit travailler, dans l'occasion, à la conservation des autres et à les garantir d'une ruine funeste, autant qu'elle peut le faire sans trop s'exposer ellemême [...] Si un peuple est désolé par la famine, tous ceux qui ont des vivres de reste doivent l'assister dans son besoin, sans toutefois s'exposer eux-mêmes à la disette [...]

\section{L'assistance, dans cette dure extrémité, est si essentiellement conforme à l'humanité} qu'on ne voit guère de nation un peu civilisée y manquer absolument [...] De quelque calamité qu'un peuple soit affligé, la même assistance lui est due $»^{4}$

Del mismo modo, puede observarse su profunda relación con la asistencia humanitaria en tiempos de guerra en la configuración jurídica que las normas de Derecho Internacional Humanitario recogían respecto de situaciones catastróficas ${ }^{5}$. Con el paso del tiempo, las normas relativas a las catástrofes acabarían alejándose del contexto bélico para formularse en tiempos de paz, y en estrecha relación con el desarrollo de sectores conexos como el medioambiente. En línea con lo anterior, puede observarse como las catástrofes son reguladas en distintos instrumentos en materia de protección

\footnotetext{
${ }^{3}$ Labor que en su origen era desarrollada cuasi exclusivamente por órdenes de tipo religioso como la Soberana Órden de Malta.

${ }^{4}$ De Vattel, E., Les Droits de Gens, ou Principes de la Loi Naturelle Appliqués à la conduite et aux affaires des Nations et des Souverains, Paris, $4^{\mathrm{a}}$ Edición, 1820 (existe una traducción al inglés: DE VATTEL, E., The Law of Nations or the Principles of Natural Law applied to the Conduct and to the Affairs of Nations and of Sovereigns, 1758, Tomos I, II, III y IV, traducción de Charles G. FENWICK con una introducción de Albert DE LAPRADELLE, Institución Carnegie de Wáshington, 1916).

${ }^{5}$ En este sentido, algunos autores han visto en el desarrollo del Derecho Internacional Humanitario un antecedente del Derecho Internacional de Catástrofes; véase CICR-OCIP, La protección civil en el Derecho Internacional Humanitario, Servicio de Asesoramiento en Derecho Internacional humanitario, Ginebra, 2001; COMISIÓN DE DERECHO INTERNACIONAL, Informe Preliminar sobre la protección de las personas en casos de desastre. Presentado por el Sr. Eduardo Valencia-Ospina, Relator Especial, doc. NN.UU. A/CN.4/598, de 5.5.2008, p. 7; FernándeZ LiESA, C.-R. y Oliva MARTíneZ, J.-D., El Derecho Internacional y la cooperación frente a los desastres en materia de Protección Civil, Dirección General de Protección Civil y Emergencias, Madrid, 2012, p. 54. De hecho, pueden encontrarse referencias en las normas del Derecho Internacional Humanitario aplicables a la protección en situaciones de catástrofe como apuntara OLIVA MARTíNEZ (vid. Íbid., p. 54) en el IV Convenio de Ginebra relativo a la protección de personas civiles en tiempo de guerra, de 12 de agosto de 1949 (UNTS vol. 75, núm. 973; BOE de 2.9.1952) o en los artículos 61 a 67 del Protocolo Adicional I a los Convenios de Ginebra de 12 de agosto de 1949, relativo a la protección de las víctimas de los conflictos armados internacionales, adoptado en Ginebra el 8 de junio de 1977 (UNTS vol. 1125, núm. 17512, BOE de 26 de julio y 7 y 9 de octubre de 1989).
} 
medioambiental, aunque limitados generalmente a las catástrofes antrópicas, es decir, aquéllas en las que interviene la mano del ser humano) $)^{6}$.

Junto con el desarrollo normativo que acaba de apuntarse, resulta de especial interés los distintos mecanismos y políticas establecidas en la gestión de catástrofes; dentro de los cuales, Naciones Unidas (ONU o NNUU) y los organismos que conforman la familia onusiana se sitúan en una posición trascendental en el plano universal.

Por tanto, como objetivo de este trabajo nos marcamos mostrar al lector una aproximación a la labor normativa e institucional de la Organización en materia de catástrofes. Con ello, pretendemos ofrecer un análisis crítico de la gestión de catástrofes por parte de Naciones Unidas que permita arrojar luz respecto de los principios y normas que regulan las catástrofes y por otro, constatar la correcta labor de la Organización acorde con su papel global en la esfera internacional.

\footnotetext{
${ }^{6}$ De este modo, pueden encontrarse tratados internacionales referidos a la responsabilidad por catástrofes de origen humano en situación de contaminación medioambiental en el Convenio sobre la Responsabilidad Civil en materia de Energía Nuclear [modificado por el Protocolo Adicional de 28 de enero de 1964, por el Protocolo de 16 de noviembre de 1982 y por el Protocolo de 12 de febrero de 2004], hecho en París el 29 de julio de 1960, entrada en vigor 1.4.1968, UNTS, vol. 956, núm. 13706, p. 251, y Anexo A en volúmenes 1041, 1056, 1144, 1155 y 1218, BOE núm. 28, de 2.2.1967; la Convención de Viena sobre Responsabilidad Civil por Daños Nucleares, aprobada el 21 de mayo de 1963, entrada en vigor el 12.11.1977, disponible en doc. OIEA INFCIRC/500, de marzo de 1996; el Convenio Internacional relativo a la Intervención en Alta Mar en Casos de Accidentes que causen o puedan causar una Contaminación por Hidrocarburos, hecho en Bruselas el 29 de noviembre de 1969, entrada en vigor 6.5.1975, UNTS vol. 970, núm. 14049, BOE núm. 49, de 26.2.1976; o el Convenio sobre la Prevención de la Contaminación del Mar por Vertimiento de Desechos y otras Materias, hecho en Londres, Méjico D. F., Moscú y Washington, el día 29 de diciembre de 1972, entrada en vigor el 30.8.1975, BOE núm. 269, de 10.11.1975. Igualmente, encontramos instrumentos con una regulación más general, aunque limitada en cuanto a su ámbito regional, pricipalmente el centroeuropeo, en el Cooperation Agreement on the Forecast, Prevention And Mitigation of Natural and Technological Disasters among the Government of the Republic of Austria, the Government of the Republic of Croatia, the Government of the Republic of Hungary, the Government of the Republic of Italy, the Government of the Republic of Poland, the Government of the Republic of Slovenia, hecho en Viena el 18 de Julio de 1992, entrada en vigor 1.8.1994, disponible en https://iea.uoregon.edu/treatytext/1992-naturaltechnologicaldisastersentxt; a sensu contrario, con una regulación sectorial pero de ámbito multilateral se establecen el Convenio de Tampere sobre el suministro de recursos de telecomunicaciones para la mitigación de catástrofes y las operaciones de socorro en caso de catástrofe, de 18 de junio de 1998, entrada en vigor 8.1.2005, UNTS, vol. 2296, núm. 40906; BOE núm. 81, de 5.4.2006; y el Convenio Marco de Asistencia en Materia de Protección Civil, de 22 de mayo de 2000, entrada en vigor 23.9.2001, UNTS, vol. 2172, núm. 38131, disponible en: https://treaties.un.org/doc/Publication/UNTS/Volume\%202172/v2172.pdf.
} 


\section{NACIONES UNIDAS Y LAS CATÁSTROFES}

Naciones Unidas constituye para muchos la organización internacional por excelencia en el panorama mundial dado la universalidad y generalidad de sus fines y que formar parte de ella la práctica totalidad de los Estados existentes en la sociedad internacional. Por ello resulta insoslayable el estudio del tratamiento que ésta ofrece en la gestión y regulación internacional de las catástrofes.

A pesar de la importancia que este tipo de fenómenos ha venido adquiriendo desde hace décadas, no se atribuyó expresamente competencias en esta materia a la ONU en el momento de elaboración de su texto fundacional, la Carta de San Francisco de $1945^{7}$. No existiendo, por tanto, una atribución explícita de competencias en materia de gestión internacional de catástrofes a Naciones Unidas $^{8}$, la fórmula empleada ha sido la de acudir a la generalidad de sus fines y a la teoría de los poderes implícitos, para permitir en la práctica una ampliación de las competencias que posee más allá de las expresamente recogidas en la Carta ${ }^{9}$. De este modo, el fundamento jurídico de la competencia de NNUU en materia de catástrofes se ha configurado con base en el artículo 1.3 de la Carta de San Francisco, y se centra en la cooperación internacional de tipo humanitaria y para el desarrollo ${ }^{10}$. Así, la principal función de la Organización se centra en «armonizar y coordinar las políticas y comportamientos estatales así como a movilizar y coordinar la prestación de asistencia humanitaria» ${ }^{11}$.

\footnotetext{
${ }^{7}$ Carta de las Naciones Unidas y Estatuto de la Corte internacional de justicia, hecha en San Francisco el 26 de junio de 1945, BOE núm. 275, de 16.11.1990 y corrección de errores en BOE núm. 285, de 28.11.1990; entrada en vigor el 24.10.1945.

${ }^{8}$ En este mismo sentido TORROJA MATEU, H. «La Organización de las Naciones Unidas ante los desastres naturales», en REQUENA HIDALGO, J. y CAMPINS ERITJA, M. (Coord.), De las catástrofes ambientales a la cotidianidad urbana: la gestión de la seguridad y el riesgo. II Coloquio Hispano-Canadiense de Barcelona, Universidad de Barcelona, Barcelona, 2000, p. 74.

${ }^{9}$ En este sentido Torroja MATEU, H. «La Organización...», op. cit., p. 74. De hecho, esta teoría de los poderes implícitos fue señalada por la propia Corte Internacional de Justicia (CIJ) desde sus primeros tiempos; vid. su Opinión Consultiva en el asunto Reparation for injuries suffered in the service of the United Nations, Advisory Opinion, ICJ Reports 1949, pp. 179-180.

${ }^{10}$ TORroja MATEU, H. «La Organización...», op. cit., p. 75.

11 TORRoJA MATEU, H., «Estrategia Internacional para la seguridad humana en los desastres naturales», en Araucaria. Revista Iberoamericana de Filosofia, Política y Humanidades, núm. 36 (2016), p. 242.
} 
La regulación de las catástrofes ha sufrido distintos avatares a lo largo de todo el siglo XX, existiendo distintos intentos por parte de la sociedad internacional de establecer una serie de normas o mecanismos para hacerlas frente. Uno de los más relevantes lo constituye el intento de la extinta la Sociedad de Naciones de establecer en 1927 una Unión Internacional de Socorro (International Relief Union) que supuso la primera organización internacional dedicada específicamente a la asistencia en caso de catástrofes $^{12}$. Tras las Grandes Guerras y con la instauración de Naciones Unidas, ésta ha servido a la regulación de las catástrofes desde sus orígenes a través de distintas vías, bien desde sus propios órganos en la adopción de normas o creación de estructuras u organismos de gestión de catástrofes, o bien sirviendo de marco en el que distintos Estados adoptarían tratados en la materia ${ }^{13}$. Otro de los remotos instrumentos elaborados para la gestión de catástrofes lo encontramos en el United Nations Relief and Rehabilitation Administration Agreement de 1943 (la Administración) ${ }^{14}$. Esta Administración se dedicaba a la provisión de asistencia en situaciones de conflicto armado y, dentro de éstas, indirectamente se ocupaba de las situaciones relacionadas con catástrofes. Sin embargo, sus funciones mucho más relacionadas con la actual protección ofrecida a los refugiados motivó que tras su disolución sus bienes se destinasen al Alto Comisionado de Naciones Unidas para los Refugiados (ACNUR) ${ }^{15}$.

\footnotetext{
${ }^{12}$ Convention and Statute establishing an International Relief Union [Convenio y Estatuto por el que se establece la Unión Internacional de Socorro], firmada en Ginebra el 12 de julio de 1927, LNTS, vol. 135, núm. 3115; entrada en vigor el 27.12.1932. Sobre ésta Organización pueden verse distintos trabajo en MACCAlister-Smith, P., «The International Relief Union», en Disasters. The International Journal of Disaster Studies and Practice, vol. 5, núm. 2 (1981), pp. 147-154; MACCALISTER-SMITH, P., «The International Relief Union: Reflections on the Convention Establishing an International Relief Union of July 12, 1927», en Legal History Review, vol. 54 (1986), pp. 363-374.

${ }^{13}$ Vid. FISCHER, H., «International disaster response law treaties: trends, patters and lacunae», en FEDERACIÓN INTERNACIONAL DE SOCIEDADES DE LA CRUZ RoJa Y DE LA MEdia Luna RoJa, International Disaster Response Laws, Principles and Practice: Reflections, Prospects and Challenges, Ginebra, 2003, p. 24. Véanse ciertas acciones en el seno de NNUU en material de asistencia a emergencias en http://www.nationsencyclopedia.com/United-Nations/Social-and-Humanitarian-AssistanceINTERNATIONAL-DISASTER-RELIEF.html.

${ }^{14}$ Hecho en Washington el 9 de noviembre de 1943, entrada en vigor el 9.11.1943 (ya no está en vigor), reproducido en United States Treaties and International Agreement 1776-1949, compiled by Charles I. Bevans, Multilateral Treaties, vol. 3 (1931-1945), Departamento de Estado, Washington, 1968, p. 845-853, disponible en https://www.loc.gov/law/help/us-treaties/bevans.php.

${ }^{15}$ Previa a la creación del ACNUR las funciones y bienes de la Administración fueron destinados a la International Refugee Organization (creada en 1946 y extinta en 1952).
} 
Las funciones que ha desarrollado NNUU en materia de catástrofes se han centrado en la promoción y cooperación en la prevención, preparación y respuesta a las catástrofes ${ }^{16}, y$ pueden agruparse en dos tipos la normativa y la institucional ${ }^{17}$. La primera se refiere a la creación de normas, reglas de conducta y principios aplicables a las catástrofes que pertenecerían al que denominamos Derecho Internacional de Catástrofes ${ }^{18}$. Mediante la segunda se establecen mecanismos, organismos o estructuras destinadas a la gestión de las catástrofes en todas o algunas de sus fases (prevención, preparación, respuesta y reconstrucción) y comprendería, entre otras, actividades de prestación de asistencia y coordinación.

Sendas funciones han sido desarrolladas en mayor o menor medida por varios de los órganos principales y subsidiarios de NNUU, adoptando diversas formas tales como Resoluciones de la Asamblea General o Informes del Secretario General ${ }^{19}$, convocatoria de conferencias mundiales en materia de reducción de catástrofes, trabajo de órganos subsidiarios como la Oficina de Coordinación de Naciones Unidas de Asuntos Humanitarios (en adelante UNOCHA) o la Comisión de Derecho Internacional (CDI), etcétera. Por tanto, no se ha centralizado en un órgano específico una u otra función. Lo anterior no ha obstado, sin embargo, a que haya sido la Asamblea General la sede que ha canalizado la mayor parte de la labor emanada de Naciones Unidas; ello en la medida en que aquélla constituye el órgano principal y motor de la Organización al establecer la política general de la misma. El motivo de este papel predominante de la Asamblea General puede encontrarse en la falta de interés de la sociedad internacional en dotarse de instrumentos con vinculación jurídica de manera generalizada, lo que en la práctica ha obligado a alcanzar avances en la materia mediante instrumentos sin vinculación jurídica ni fuerza coercitiva como son las resoluciones de la Asamblea General ${ }^{20}$.

\footnotetext{
${ }^{16}$ Vid. entre otros, TORROJA MATEU, H., «Estrategia...» op. cit., pp. 247-252.

${ }^{17}$ Funciones que autores como TORROJA MATEU denomina poderes normativo y de acción; vid. TORROJA MATEU, H. «La Organización...», op. cit., p. 76.

${ }^{18}$ Siguiendo la noción que ofrecemos de Derecho Internacional de Catástrofes, se entiende por éste aquel sector del ordenamiento jurídico internacional destinado a regular el fenómeno de las catástrofes y las actividades relacionadas con su gestión en todas sus fases, con el fin de proteger a las personas, sus bienes o el medioambiente.

${ }_{19}$ Pueden verse las Resoluciones e Informes en materia de catástrofes en el siguiente enlace: http://www.unisdr.org/we/inform/resolutions-reports.

${ }^{20}$ Según el tenor literal de la Carta de San Francisco, sólo gozarían de fuerza vinculante para sus miembros las Resoluciones del Consejo de Seguridad en virtud del artículo 25 que establece que «Los Miembros de
} 
En este sentido, junto con la Asamblea General, el Consejo Económico y Social (en adelante ECOSOC) y la Secretaría General, participan en la gestión y regulación internacional de las catástrofes las distintas agencias especializadas, programas y fondos que conforman la familia onusiana ${ }^{21}$. Además, junto con la labor específica de las distintas agencias u órganos, la reducción del impacto provocado por las catástrofes naturales es considerada una tarea transversal a toda la Organización en el desarrollo diario de sus cometidos $^{22}$. A continuación se muestra un cuadro que refleja las entidades con funciones en materia de catástrofes más relevantes dentro de la organización.

\section{SISTEMA DE NACIONES UNIDAS EN MATERIA DE CATÁSTROFES}

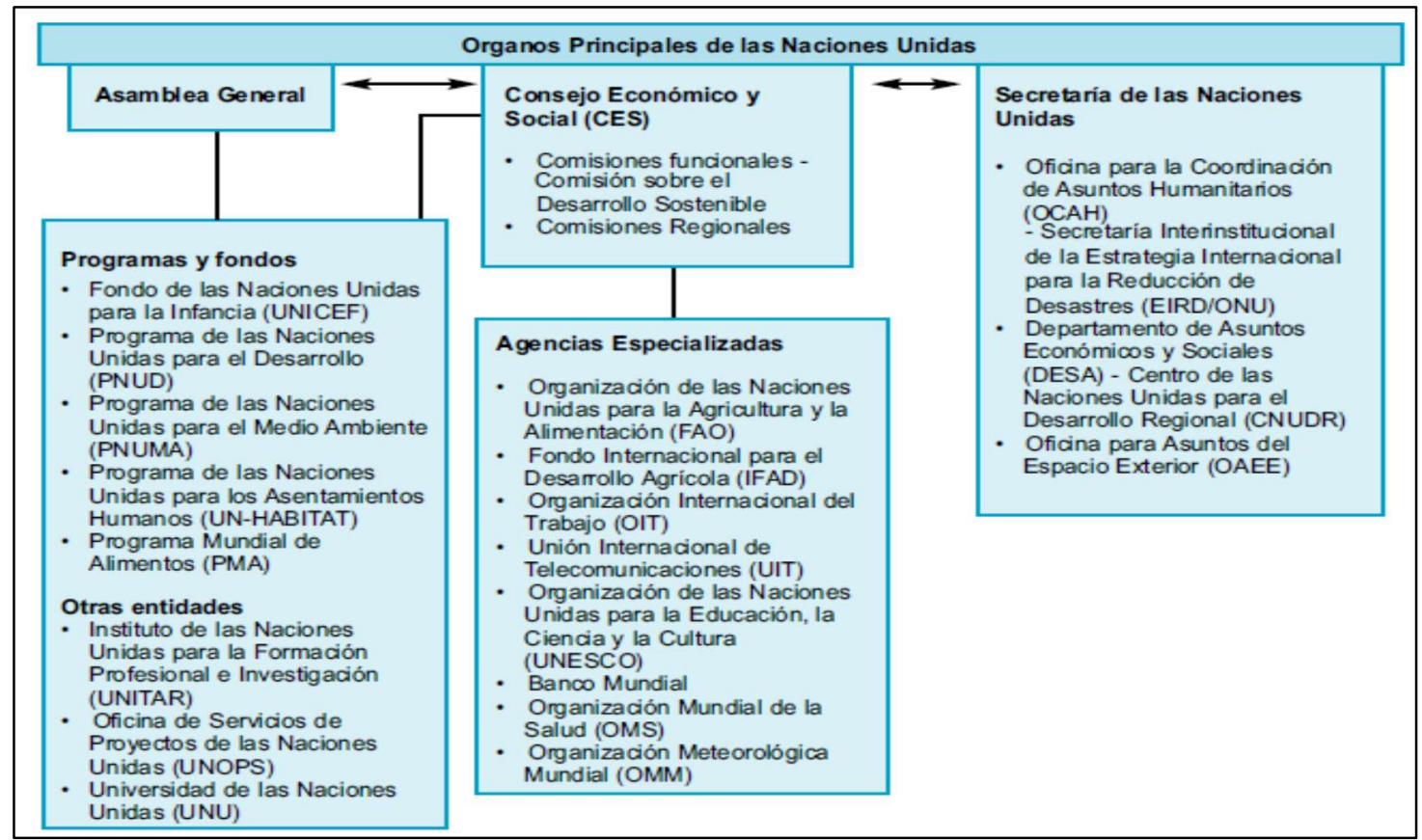

las Naciones Unidas convienen en aceptar y cumplir las decisiones del Consejo de Seguridad de acuerdo con esta Carta».

${ }^{21}$ Vid. OKERE, B. y MAKAWA, E.-M., «Global Solidarity and the International Response to Disasters», en CARON, D.-D. y LEBEN, C. (Eds.), Les aspects internationaux des catastrophes naturelles et industrielles/The international aspects of natural and industrial catastrophes, The Hague Academy of International Law, Martinus Nijhoff, The Hague/Boston/London, 2001, pp. 429-456. Y en una información más actual en UNISDR, Disaster Risk Reduction in the United Nations. Roles, mandates and results of Key UN entities, 2013. Respecto del trabajo realizado por la UNESCO en materia de catástrofes puede verse BAutista-HernáEZ, A., «Reflexiones en torno a la Regulación Jurídica de la Reducción del Riesgo de Desastre (Disaster Risk Reduction) y la Práctica Internacional al respecto seguida por la UNESCO», en Ius et Scientia, Vol. 2, núm. 1 (2016), pp. 89-107.

${ }^{22}$ Vid. al respecto ESTRATEGIA INTERNACIONAL PARA LA REDUCCIÓN DE DESASTRES / NACIONES UNIDAS, Vivir con el riesgo: informe mundial sobre iniciativas para la reducción de los desastres, Edita Naciones Unidas, Nueva York y Ginebra, 2004, Anexo 3, p. 74. 
Fuente: ESTRATEGIA INTERNACIONAL PARA LA REDUCCIÓN DE DESASTRES / NACIONES UNIDAS, Vivir con el riesgo: informe mundial sobre iniciativas para la reducción de los desastres, Edita Naciones Unidas, Nueva York y Ginebra, 2004, Anexo 3, p. 75.

\section{Aspecto Institucional}

La creación de instrumentos, organismos subsidiarios o procedimientos para la coordinación internacional en caso de catástrofes corresponde a la Asamblea General y al ECOSOC ${ }^{23}$. Éste último, bajo la autoridad de la Asamblea General,

«...contribute to strengthening the coordination and effectiveness of UN humanitarian assistance and support and complement international efforts aimed at addressing humanitarian emergencies, including natural disasters, in order to promote an improved, coordinated response by the $\mathrm{UN} \gg^{24}$.

Además, las Resoluciones de la Asamblea General van a jugar un papel muy relevante a nivel organizativo, pues van a ser el vehículo en las que por mandato de la Asamblea al Secretario General se creen organismos subsidiarios tales como Oficinas, Planes o Fondos, como ocurrió con la creación de la antecesora de la UNOCHA, la Oficina del Coordinador de NNUU del Socorro para Casos de Desastres (UNDRO) a través de la Resolución 2816 (XXVI), de 14 de diciembre de $1971^{25}$.

Naciones Unidas no obtuvo una presencia relevante en la respuesta a las catástrofes en la escena internacional hasta la década de 1960. Hasta entonces sólo se había contado con una participación limitada de algunos de sus organismos especializados en materia de refugiados, alimentación, salud o cultura. Dada la

\footnotetext{
${ }^{23}$ Véase al respecto TORROJA MATEU, H. «La Organización...», op. cit., pp. 75, 77-78. La posibilidad de creación de órganos subsidiarios aparece recogida en los artículos 7.2, 29, 68 y 107 de la Carta de San Francisco, y específicamente referidos a la Asamblea General en el artículo 22.

${ }^{24}$ Vid. United Nations Handbook 2017-18, publicado por el Ministerio de Asuntos Exteriores de Nueva Zelanda, 2017 (reeditada anualmente desde 1961), disponible en https://www.mfat.govt.nz/assets/Peaceand-Security/United_Nations_Handbook_2017_18.pdf., p. 141.

${ }^{25}$ Titulada Asistencia en casos de desastres naturales y otras situaciones de desastre, doc. NNUU $\mathrm{A} / \mathrm{RES} / 2816(\mathrm{XXVI})$.
} 
multiplicidad de organizaciones internacionales intergubernamentales y no gubernamentales que participaban en las actividades de asistencia, se hizo patente la necesidad de establecer mecanismos de coordinación a nivel internacional para centralizar la respuesta ofrecida y canalizar los recursos financieros puestos por la sociedad internacional o instituciones privadas a tal fin ${ }^{26}$. Para ello se crea en 1971 un Coordinador del Socorro para Casos de Desastre y su Oficina (la UNDRO). El Coordinador con la categoría de Secretario General Adjunto, fue establecido en un principio por un período de 5 años, aunque la necesidad de actuación debido a las numerosas catástrofes motivó la ampliación de su mandato ${ }^{27}$. Los nuevos desafíos de la asistencia humanitaria así como el aumento en el número y consecuencias de las catástrofes motivarían distintas reformas en el organigrama del sistema para el fortalecimiento de la asistencia humanitaria ${ }^{28}$ que darían lugar a la creación de la UNOCHA y previamente en 1991 del Comité Permanente entre Organismos o Comité Permanente Interinstitucional, (conocido por sus siglas en ingles IASC) ${ }^{29}$.

De este modo, los principales instrumentos creados en el seno de NNUU para la gestión internacional de las catástrofes, y que permanecen en la actualidad, son: la Oficina

\footnotetext{
${ }^{26}$ En este sentido vid. ThouvenIN, J.-M., «L'internationalisation des secours en cas de catastrophe naturelle», en Revue General de Droit International Publique, Tomo 102, vol. 2 (1998), pp. 340-341.

${ }^{27}$ Desde su creación se ha recordado la utilidad y necesidad de mejora de la UNDRO en numerosas resoluciones de la Asamblea General; vid. Resolución 3243 (XXIX) de 29 de noviembre de 1974, Fortalecimiento de la Oficina del Coordinador de las Naciones Unidas para el Socorro en caso de Desastre, doc. NNUU A/RES/3243(XXIX); Resolución 34/55, de 29 de noviembre de 1979, Oficina del Coordinador de las Naciones Unidas para el Socorro en Casos de Desastre, doc. NNUU A/RES/34/55; Resolución 36/225, de 17 de diciembre de 1981, Fortalecimiento de la capacidad del sistema de las Naciones Unidas para responder en situaciones de desastres naturales y de otra índole, doc. NNUU A/RES/36/225; Resolución 37/144, de 17 de diciembre de 1982, Oficina del Coordinador de las Naciones Unidas para el Socorro en Casos de Desastre, doc. NNUU A/RES/34/144; Resolución 39/207, de 17 de diciembre de 1984, Oficina del Coordinador de las Naciones Unidas para el Socorro en Casos de Desastre, doc. NNUU A/RES/39/207; Resolución 41/201, de 8 de diciembre de 1986, Oficina del Coordinador de las Naciones Unidas para el Socorro en Casos de Desastre, doc. NNUU A/RES/41/201; Resolución 45/221, de 21 de diciembre de 1990, Fortalecimiento de la Oficina del Coordinador de las Naciones Unidas para el Socorro en Casos de Desastre, doc. NNUU A/RES/45/221.

${ }^{28}$ En este sentido THOUVENIN, J.-M., «L'internationalisation...», op. cit., p. 342.

${ }^{29}$ Tras la Resolución 46/182, de 19 de diciembre de 1991, de la Asamblea General, se establece el IASC y posteriormente el Departamento de Asuntos Humanitarios (DHA) que sustituiría a la UNDRO. Tras la reforma de la Secretaría en 1998 (vid. Renovación de las Naciones Unidas: Un programa de reforma, Informe del Secretario General, doc. NN.UU. A/51/950, de 14.7.1997, párr. 77), se reestructura el DHA que será sustituido por la UNOCHA.
} 
de Coordinación de Naciones Unidas de Asuntos Humanitarios y el Comité Permanente entre Organismos ${ }^{30}$.

\section{A) COORDINACIÓN HUMANITARIA: LA OFICINA DE COORDINACIÓN DE NACIONES UNIDAS DE ASUNTOS HUMANITARIOS}

La UNOCHA fue creada por Resolución de la Asamblea General 46/182, de 19 de diciembre de 1991 y depende orgánicamente de la secretaría general. Su papel consiste en asegurar una puesta en común de las organizaciones encargadas de la asistencia humanitaria para asegurar una respuesta coherente mediante la movilización y coordinación de las actividades humanitarias, la defensa de los derechos de las personas necesitadas y la promoción de la prevención y preparación ${ }^{31}$. Sus funciones principales pueden enumerarse en cinco categorías: coordinación humanitaria, desarrollo de políticas, promoción, financiación e información ${ }^{32}$. Su misión se enmarca dentro del objetivo global por parte de Naciones Unidas de centralizar los esfuerzos de la sociedad internacional en asistir a los Estados afectados por una catástrofe ${ }^{33}$.

La UNOCHA elabora periódicamente un Plan Estratégico en el que establece sus objetivos generales y estratégicos. En el correspondiente al período $2014-2017^{34}$, se pone el foco en la mejora de la ayuda humanitaria así como en la efectividad de la respuesta a las crisis por las organizaciones humanitarias.

En materia presupuestaria, la UNOCHA financia el mantenimiento de sus oficinas principales con cargo al presupuesto ordinario de la Organización, así como con un 7\% del fondo extrapresupuestario (según presupuesto para $2017^{35}$ ) y sus actividades en el

\footnotetext{
${ }^{30}$ Cuya denominación oficial en inglés es Inter-Agency Standing Committee.

${ }^{31}$ Vid. http://www.unocha.org/about-us/who-we-are.

${ }^{32}$ United Nations Handbook 2017-18... op .cit., p. 221.

${ }^{33}$ Vid. Resolución 46/182, párr. 12.

34 Vid. OCHA Strategic Plan 2014-2017, disponible en http://www.unocha.org/sites/unocha/files/dms/Documents/OCHA\%20SF\%2020142017\%20Strategic\%20Plan.pdf.

35 Vid. OCHA Plan \& Budget 2017, marzo de 2017, disponible en http://www.unocha.org/sites/unocha/files/OCHA_P_B_2017.pdf, p. 23.
} 
terreno mediante éste último integrado por fondos fiduciarios financiados mediante aportaciones voluntarias. La mayor parte del presupuesto de la UNOCHA se destina a la realización de sus objetivos y se nutre de aportaciones voluntarias de los Estados y Organizaciones internacionales. Sin embargo, este presupuesto extraordinario se ha visto reducido para 2017 en un 19 por 100 (49 millones de dólares), por lo que sigue imperando la tónica general según la cual pese al apoyo político de que goza la Oficina, no ocurre lo mismo en la aportación de fondos, lo cual ha propiciado constantes obstáculos en la consecución de sus objetivos ${ }^{36}$.

\section{B) COORDINACIÓN DENTRO Y FUERA DEL SISTEMA DE NNUU: EL COMITÉ PERMANENTE ENTRE ORGANISMOS}

El IASC es un instrumento interinstitucional establecido en mediante la Resolución de la Asamblea General 46/182, de 19 de diciembre de $1991^{37}$, para coordinar la respuesta a las situaciones de emergencia por parte de las organizaciones operacionales.

El Coordinador del Socorro de Emergencia de las Naciones Unidas preside el IASC. Pueden formar parte del Organismo todas las organizaciones operacionales incluyendo tanto Organizaciones Intergubernamentales como ONG, y tienen invitación permanente para participar el Comité Internacional de la Cruz Roja, la Liga de Sociedades de la Cruz Roja y de la Media Luna Roja y la Organización Internacional para las Migraciones. En la práctica de las reuniones no existe distinción entre los miembros con invitación permanente y los no permanentes ${ }^{38}$.

La relación entre la UNOCHA y el IASC en una situación de catástrofe es la siguiente. Cuando se produce una emergencia (catástrofe natural, antrópica o mixta) el máximo órgano responsable de la coordinación de la respuesta por las organizaciones es la UNOCHA, la cual ser sirve del IASC para la coordinación de las distintas entidades

\footnotetext{
${ }^{36}$ En esta misma opinión, vid. TORROJA MATEU, H., «Estrategia...» op. cit., p. 251.

${ }^{37}$ Anexo, párr. 38. NACIONES UnIDAS, The United Nations Today, Departamento de Información Pública, Nueva York, 2008, p. 266.

${ }^{38}$ Vid. United Nations Handbook 2017-18... op .cit., p. 223.
} 
del sistema onusiano y fuera de éste ${ }^{39}$. El papel principal del IASC es, por tanto, servir como instrumento cardinal en la coordinación de la asistencia humanitaria entre los organismos que la prestan tanto dentro como fuera del sistema de Naciones Unidas. Igualmente, es un foro de desarrollo de políticas y reglas para los agentes humanitarios. Periódicamente el IASC establece una serie de prioridades temáticas, habiendo acordado en 2017 las siguientes: respuesta efectiva a emergencias y crisis duraderas, rendición de cuentas e inclusión, desplazamientos y financiación ${ }^{40}$.

\section{Aspecto Normativo}

Respecto del rol normativo, es decir, el establecimiento de normas aplicables a situaciones de catástrofe, será la Asamblea General la que juegue un papel preeminente en el seno de Naciones Unidas. Ésta actúa mediante distintas acciones como la elaboración y el fomento en la adopción de tratados internacionales ${ }^{41}$; la organización de distintas conferencias mundiales centradas en la reducción del riesgo de catástrofe ${ }^{42}$, así como las reuniones de alto nivel organizadas normalmente en paralelo a las sesiones de

\footnotetext{
${ }^{39}$ Este papel de coordinador ha sido recogido, por ejemplo, en el Mecanismo de Protección Civil de la Unión establecido por la Decisión 1313/2013/UE, del Parlamento Europeo y del Consejo, de 17 de diciembre de 2013, relativa a un Mecanismo de Protección Civil de la Unión, DOUE L 347/924, de 20.12.2013. En este Mecanismos se reconoce el papel de coordinación general que debe desarrollar la UNOCHA (vid. artículo 16 de la Decisión). Puede verse un análisis sobre la misma en BAUTISTA-HERNÁEZ, A., «Recientes avances en la regulación de la Unión Europea en materia de catástrofes: el Mecanismo de Protección Civil de la Unión», en García SAN José, D., SÁnChEZ Patrón, J.-M. y TORRES CaZORLA, M.I. (Coords.), Bioderecho, Seguridad y Medioambiente. Biolaw, Security and Environment, Tirant lo Blanch, Valencia, 2015, pp. 173-194.

${ }^{40}$ Vid. United Nations Handbook 2017-18... op .cit., p. 222.

${ }^{41}$ Como el intento de Convención realizada en el marco de la UNDRO en 1984, que marcó un punto de inflexión referido a las distintas reglas que podría contener un tratado de esta naturaleza (vid. COMISIÓN DE DERECHO INTERNACIONAL, Informe Preliminar sobre la protección de las personas en casos de desastre. Presentado por el Sr. Eduardo Valencia-Ospina, Relator Especial, doc. NNUU A/CN.4/598, de 5.5.2008, p. 15); o la Convención internacional de lucha contra la desertificación en los países afectados por sequía grave o desertificación, en particular en África, doc. NNUU A/AC.241/24, de 12.9.1994.

${ }_{42}$ Así, entre muchas otras, la Cumbre Humanitaria Mundial, en cuyos documentos preparatorios se reclamaba que se tuviese en cuenta a la población afectada en caso de desastre, que los gobiernos estableciesen convenios internacionales en materia de preparación y respuesta para amenazas naturales con la comunidad internacional; vid. WORLD HUMANITARIAN SUMMIT SECRETARIAT, Restoring Humanity: Synthesis of the Consultation Process for the World Humanitarian Summit, New York, United Nations, 2015, disponible en: http://reliefweb.int/sites/reliefweb.int/files/resources/Restoring\%20Humanity\%20Synthesis $\% 20$ of $\% 20$ the $\% 20$ Consultation $\% 20$ Process $\% 20$ for $\% 20$ the $\% 20$ World $\% 20$ Humanitarian $\% 2$ 0Summit.pdf.
} 
la Asamblea General ${ }^{43}$; y principalmente, mediante la adopción de Resoluciones y los trabajos de codificación y desarrollo progresivo (en el seno de su Comisión de Derecho Internacional) ${ }^{44}$.

Igualmente, debe destacarse la proclamación en su Resolución 44/236, de 22 de diciembre de 1989 del Decenio Internacional para la reducción de los desastres naturales (DIRDN) y el Marco Internacional de Acción ${ }^{45}$. Aquél tiene por objetivo

«Reducir, por medio de una acción internacional concertada, especialmente en los países en desarrollo, la pérdida de vidas, los daños materiales y las perturbaciones sociales y económicas causadas por desastres naturales como terremotos, vendavales (ciclones, huracanes, tornados, tifones), maremotos, inundaciones, desprendimientos de tierra, erupciones volcánicas, incendios y otras calamidades de origen natural, tales como la plaga de saltamontes y de la langosta ${ }^{46}$.

La idea que comenzó aquí y que perdura en las iniciativas sobre reducción de catástrofes hasta nuestros días es la de cambio de paradigma, desde la imposibilidad de actuar frente a las catástrofes naturales hasta el conocimiento de que sí puede disminuirse el riesgo de que se produzcan. De ahí que desde estas épocas se comenzase a poner el

\footnotetext{
${ }^{43}$ Entre las que destacamos la Reunión plenaria de alto nivel de la Asamblea General en su $60^{\circ}$ período de sesiones «Cumbre Mundial 2005» celebrada del 14 al 16 de septiembre de 2005, en la Sede de las Naciones Unidas, Nueva York (véase sobre ésta http://www.un.org/spanish/summit2005/) y en la que se adoptaron compromisos en materia de asistencia en caso de catástrofes, en concreto la creación del Fondo central para la acción en casos de emergencia (CERF, por sus siglas en inglés). El Central Emergency Response Fund fue creado en marzo de 2006 como mejora del instrumento de financiación previo Central Emergency Revolving Fund. El CERF es un mecanismo integrado en la UNOCHA para la recepción de donaciones provenientes de entidades públicas o privadas; tiene el objetivo de recaudar 450 millones de dólares anuales que son empleados en la respuesta inmediata a emergencias. Sobre el fondo véanse NACIONES UNIDAS, The United Nations Today, Departamento de Información Pública, Nueva York, 2008, pp. 17 y 268; la aprobación por la Asamblea General de Naciones Unidas de los compromisos de la cumbre en su Resolución 60/1, de 16 de septiembre de 2005, Documento Final de la Cumbre Mundial 2005, doc. NNUU A/RES/60/1, de 24.10.2005; o su página web http://www.unocha.org/cerf/.

${ }^{44}$ Vid. TORROJA MATEU, H., «Estrategia...» op. cit., p. 253.

${ }^{45}$ Resolución 44/236, de 22 de diciembre de 1989, Decenio Internacional para la Reducción de los Desastres Naturales, doc. NNUU A/RES/44/236. Proclamación que ya vendría auspiciada por designación en 1987 del decenio 1990 para «la especial atención al fomento de la cooperación internacional en la esfera de la reducción de los desastres naturales; vid. Resolución 42/169, de 11 de diciembre de 1987, Decenio Internacional para la Reducción de los Desastres Naturales, doc. NNUU A/RES/42/169, apartado 3.

${ }^{46}$ Resolución 42/169, de 11 de diciembre de 1987, apartado 4.
} 
foco en las actividades en materia de prevención y no tanto en las de respuesta una vez producida la catástrofe.

La Conferencia Internacional de Yokohama (Japón) de 1994 se celebró para evaluar las metas alcanzadas por el Decenio en el meridiano de su mandato y proponer mejoras de actuación. En el Informe final aprobado en la Conferencia se contiene la Estrategia de Yokohama para un mundo más seguro: directrices para la prevención de los desastres naturales, la preparación para casos de desastre y la mitigación de sus efectos en que figuran los principios, la estrategia y el plan de acción ${ }^{47}$, en la que se proponen como líneas estratégicas, principalmente: 1) propiciar un mayor uso de las ciencias sociales en lugar de las ciencias naturales que venían encabezando la visión científica en la elaboración de políticas; 2) la mejora de las normas internas de los Estados en materia de reducción del riesgo de catástrofe; 3) el fortalecimiento de redes regionales para la cooperación técnica y el intercambio de información; 4) cambio en el enfoque de las medidas de prevención, desde aquéllas centradas en los preparativos de emergencia hacia las enfocadas en la reducción de los factores de vulnerabilidad y riesgo.

Al final de la década se llevarían a cabo sendos encuentros con el objetivo de discutir sobre el futuro del Decenio: por un lado en el seno del ECOSOC, y por otro el Foro Programático del Decenio Internacional para la Reducción de los Desastres Naturales celebrado en Ginebra del 5 al 9 de julio de 1999. Todo ello junto con otras reuniones a nivel regional ${ }^{48}$. Al término del DIRDN, la continuación en el desarrollo

\footnotetext{
${ }^{47}$ Informe de la Conferencia Mundial sobre la Reducción de los Desastres Naturales (Yokohama, 23 a 27 de mayo de 1994), doc. NNUU A/CONF.172/9, de 27.9.1994, Resolución 1, Anexo I.

${ }^{48}$ Véase v. gr. las reuniones en Europa (Praga, junio de 1998 y Londres, junio de 1998); la Séptima reunión anual regional del Pacífico Sur sobre desastres (Nuku'alofa, Tonga, julio de 1998); la Conferencia regional para la CEI y los países de Europa central y oriental (Yerevan, septiembre de 1998); la Reunión regional DIRDN-CESPAP para Asia (Bangkok, febrero de 1999); Prevención y reducción de desastres naturales en el Mediterráneo (Valencia, mayo de 1999); la Reunión regional DIRDN-PNUMA para África (Nairobi, mayo de 1999); la Reunión Hemisférica del DIRDN: Hacia la Reducción de los Desastres en el Siglo XXI en las Américas (San José, Costa Rica del 31 de mayo al 5 de junio de 1999); así como un conjunto de reuniones temáticas como la Reunión intergubernamental sobre telecomunicaciones en casos de emergencia (Tampere, Finlandia, junio de 1998); la Conferencia internacional sobre alerta temprana (Potsdam, Alemania, septiembre de 1998); el Seminario internacional sobre reducción de desastres naturales en las carreteras en los países mediterráneos (Estambul, octubre de 1998); la Primera reunión intergubernamental de expertos sobre El Niño (Guayaquil, Ecuador, noviembre de 1998); la Conferencia sobre peligros naturales de montaña (Grenoble, abril de 1999); y la conferencia sobre Prevención de desastres naturales: planificación del uso de la tierra y desarrollo sostenible (París, junio de 1999).
} 
normativo y político en materia de catástrofes vendría con la adopción de la Estrategia Internacional de Reducción de Desastres (EIRD), establecida por la Resolución 54/21949, así como el Marco de acción para su aplicación ${ }^{50}$. Tras una evaluación de los frutos del Decenio, la Estrategia creada debería:

«a) Proporcionar las condiciones óptimas para garantizar que la promoción de la reducción de desastres tenga un carácter consultivo e interinstitucional; b) Facilitar el diálogo permanente con los ámbitos de la asistencia humanitaria y el desarrollo sostenible y fomentar las sinergias y la complementariedad entre ambas; y c) Dar preeminencia a los objetivos de reducción de los desastres dentro del marco de las Naciones Unidas ${ }^{51}$.

Igualmente, la EIRD desarrolla distintos aspectos relacionados con la prevención en caso de catástrofe. De este modo, a nivel conceptual, destaca la creación de un grupo de trabajo formado por expertos internacionales sobre terminología (el OIEWG), cuya primera reunión formal tuvo lugar en Ginebra los días 29-30 de septiembre de $2015^{52}$ y cuyos trabajos finalizaron en $2016^{53}$.

Junto con la Estrategia se establece una Secretaría Interinstitucional de la Estrategia Internacional para la Reducción de los Desastres, más adelante conocida como Oficina de NNUU para la Reducción del Riesgo de Desastre (UNISDR, por sus siglas en inglés) con el objetivo de servir de punto focal en materia de reducción de catástrofes naturales en el sistema de NN.UU., así como desarrollar la cooperación con las

\footnotetext{
${ }^{49}$ Resolución 54/219, de 22 de diciembre de 1999, Decenio Internacional para la Reducción de los Desastres Naturales: nuevas disposiciones, doc. NNUU A/RES/54/219, de 3.2.2000. Completada con la Resolución 56/195, de 21 de diciembre de 2001, Estrategia Internacional de Reducción de Desastres, doc. NNUU A/RES/56/195, de 21.1.2002. Igualmente, mediante la Resolución 54/219 se crearía el Equipo de Tareas Interinstitucional sobre Reducción de Desastres (párr. 4).

${ }^{50}$ Contenido en Aplicación de la Estrategia Internacional de Reducción de Desastres, Informe del Secretario General, doc. NNUU A/56/68-E/2001/63, de 8.5.2001 y Corr.1.

${ }^{51}$ Recomendaciones sobre los arreglos institucionales para las actividades de reducción de desastres del sistema de las Naciones Unidas después de la conclusión del Decenio Internacional para la Reducción de los Desastres Naturales, Informe del Secretario General, doc. NNUU A/54/136-E/1999/89, de 18.6.1999, parr. 24, p. 13.

${ }_{52} \mathrm{Vid}$. http://www.preventionweb.net/drr-framework/open-ended-working-group/.

${ }^{53}$ Con la adopción de las Recomendaciones del grupo de trabajo intergubernamental de expertos de composición abierta sobre los indicadores y la terminología relacionados con la reducción del riesgo de desastres, en Informe del grupo de trabajo intergubernamental de expertos de composición abierta sobre los indicadores y la terminología relacionados con la reducción del riesgo de desastres, apartado V., doc. NNUU A/71/644, de 1.12.2016 (y A/71/644/Corr.1).
} 
organizaciones regionales ${ }^{54}$. El fin de la secretaría sería «la promoción, la formulación de políticas y la coordinación de las actividades del sistema de las Naciones Unidas en el ámbito de la reducción de desastres naturales» ${ }^{55}$.

La Segunda Comisión de la Asamblea General examina periódicamente en el tema relativo al desarrollo sostenible la Estrategia Internacional para la Reducción de Desastres $^{56}$, recomendando al plenario la adopción de una resolución sobre reducción del riesgo de desastre. La Asamblea General ha seguido analizando la cuestión en las sucesivas sesiones ${ }^{57}$. Además, vio con agrado las adopciones del Marco de Acción de Hyogo 2005-2015 58 , y posteriormente del Marco de Sendai para la Reducción del Riesgo de Desastre 2015-2030 ${ }^{59}$. Éste último exige una acción coordinada de la familia de NNUU y las acciones de los Estados Miembros, mediante un uso eficiente de los recursos ${ }^{60}$. Desde entonces las políticas sobre reducción de catástrofes se encaminan en consonancia con el Marco de Sendai y se centran en su aplicación ${ }^{61}$. Desde su $71^{\circ}$ período de sesiones

\footnotetext{
${ }^{54}$ Vid. la Resolución 56/195, de 21 de diciembre de 2001, párr. 6.

${ }^{55}$ Ello a través de las siguientes funciones: «a) Actuar dentro del sistema de las Naciones Unidas como el centro de coordinación de estrategias y programas de reducción de desastres naturales y asegurar las sinergias entre las estrategias de reducción de los desastres naturales y las que se aplican en los ámbitos económicos y humanitarios; b) Apoyar al grupo de trabajo interinstitucional en la formulación de políticas de reducción de desastres naturales; c) Hacer comprender en todo el mundo la necesidad de reducir los efectos negativos de los peligros naturales por medio de campañas de promoción; d) Actuar como una centro internacional de difusión e intercambio de información y conocimientos sobre estrategias de reducción de desastres; e) Respaldar las políticas y actividades de promoción de los comités nacionales para la reducción de desastres naturales»; vid. Recomendaciones sobre los arreglos institucionales para las actividades de reducción de desastres del sistema de las Naciones Unidas después de la conclusión del Decenio Internacional para la Reducción de los Desastres Naturales, Informe del Secretario General, doc. NNUU A/54/136-E/1999/89, de 18.6.1999, párr. 21, p. 7.

${ }^{56}$ Véase el programa de trabajo de ésta para el $71^{\circ}$ período de sesiones de la A.G. en el doc. NNUU A/C.2/70/L.1, de 8.9.2015; Programa definitivo del septuagésimo segundo período ordinario de sesiones de la Asamblea Genera se encuentra en el doc. NNUU A/72/251, de 15.9.2017.

57 Véanse las Resoluciones de la Asamblea General 56/194, 56/195, 57/255, 57/256, 58/214, 58/215, 59/231, 59/232, 59/233, 60/195, 60/196, 61/198, 61/199, 61/200, 62/192, 63/215, 63/216, 63/217, 64/200, 65/157, 65/158, 66/199, 67/208, 67/209, 68/99, 68/211, 69/219, 69/283, 69/284, 70/110, 70/203 y 70/204. ${ }^{58}$ Declaración de Hyogo y el Marco de Acción de Hyogo para 2005-2015: Aumento de la Resiliencia de las Naciones y las Comunidades ante los Desastres, aprobados por la Conferencia Mundial sobre la Reducción de los Desastres, celebrada en Kobe (Hyogo, Japón) del 18 al 22 de enero de 2005 (Resolución 60/195)

${ }^{59}$ Declaración de Sendái y el Marco de Sendái para la Reducción del Riesgo de Desastres 2015-2030, aprobados por la Tercera Conferencia Mundial de las Naciones Unidas sobre la Reducción del Riesgo de Desastres, celebrada en Sendái (Japón) del 14 al 18 de marzo de 2015 (Resolución 69/283).

${ }^{60}$ Vid. Aplicación del Marco de Sendai para la Reducción del Riesgo de Desastres 2015-2030, Informe del Secretario General, doc. NNUU A/71/230, de 29.7.2016, párr. 23, p. 8.

${ }^{61}$ Sobre la aplicación del Marco de Sendai resultan de interés los distintos Informes del Secretario General al respecto, siendo el más reciente de julio de 2016; vid. Aplicación del Marco de Sendai para la Reducción del Riesgo de Desastres 2015-2030, Informe del Secretario General, doc. NNUU A/71/230, de 29.7.2016.
} 
los esfuerzos de la Asamblea General se canalizan en la implantación del Plan de Acción de las Naciones Unidas sobre la Reducción del Riesgo de Desastres para la Resiliencia: "Hacia un enfoque integrado del desarrollo sostenible que tenga en cuenta los riesgos" vinculado con la Agenda $2030^{63}$ y el Acuerdo de París.

En otro orden de cosas, debemos dedicar algunas líneas a la consideración jurídica de las normas emanadas en el seno de la Asamblea General. Como hemos apuntado más arriba, estas normas pertenecerían al sector jurídico denominado Derecho Internacional de Catástrofes (también conocido como Derecho Internacional de los desastres ${ }^{64}$, Droit des catastrophes ${ }^{65}$, o Derecho Internacional de las Intervenciones en caso de Desastres ${ }^{66}$ o International Law of Disasters ${ }^{67} \mathrm{o}$ International Disaster Response Law ${ }^{68}$ ). El problema que se plantea aquí se refiere a la fuerza coercitiva u obligatoriedad jurídica de las normas emanadas en el seno de la Asamblea General de Naciones Unidas. Existe unanimidad por parte de la doctrina iusinternacionalista más autorizada en no otorgar un valor jurídico vinculante a las Resoluciones de la Asamblea General en base al tenor de la Carta de San Francisco, quien atribuye exclusivamente tal valor a las Resoluciones del Consejo de Seguridad. No obstante, y puesto que atendiendo al tratado constitutivo de la organización, esta puede o no atribuir un valor jurídico para sus Estatos miembros, no son pocos los autores que entienden la posibilidad de atribuir un «efecto jurídico extrínseco» a estas Resoluciones. De este modo, aquellas resoluciones de una Organización que se

\footnotetext{
${ }^{62}$ Vid. Aplicación del Marco de Sendai para la Reducción del Riesgo de Desastres 2015-2030, Informe del Secretario General, doc. NNUU A/71/230, de 29.7.2016, pp. 8-10.

${ }^{63}$ Establecida en la Resolución 70/1, Transformar nuestro mundo: la Agenda 2030 para el Desarrollo Sostenible, de 25 de septiembre de 2015, doc. NNUU S/RES/70/1, de 21.10.2015.

${ }^{64}$ Empleado por TORROJA MATEU, H., «Estrategia...» op. cit., p. 253.

${ }^{65}$ LiEnHARD, C., «Pour un droit des catatrophes», Recueil Dalloz, núm. 13, 30 de marzo de 1995, recurso electrónico, n.p. (la versión impresa comprende pp. 91-102).

${ }^{66}$ Así recogido, entre otros, en NACIONES UNIDAS, Informe de la Comisión de Derecho Internacional sobre la labor realizada en su quincuagésimo octavo periodo de sesiones, publicado como Documentos Oficiales de la Asamblea General, sexagésimo primer periodo de sesiones, Suplemento $N^{o} 10$, doc. NN.UU. A/61/10, de 2006, p. 524.

${ }^{67}$ Empleado en la obra de CARON, D.-D., et al. (ed.), The International Law of Disaster Relief, Cambridge University Press, Cambridge, 2014.

${ }^{68}$ Empleado por HOFFMAN, M.-H., «What is the Scope of International Disasters Response Law?», en FEDERACIÓN INTERNACIONAL DE SOCIEDADES DE LA CRUZ RoJA Y DE LA MEDIA LunA ROJA, International Disaster Response Laws, Principles and Practice: Reflections, Prospects and Challenges, Ginebra, 2003, p. 13; GutTRY, A. DE, Gestri, M., VenturinI, G. (Eds.), International Disaster Response Law, TMC Asser Press, The Hague (The Netherlands), 2012; así como en los numerosos trabajos en el seno de la Cruz Roja y el Proyecto International Disaster Response Law Programme.
} 
manifiesten respecto del Derecho internacional general y no limitándose a los principios contenidos en su tratado constitutivo

«podrá[n] operar como elemento en el proceso de formación de la costumbre [...] para determinar la opinio iuris de los Estados que han participado en su adopción» ${ }^{69}$.

Por tanto, deberemos acudir a la casuística en la adopción de las distintas Resoluciones de la Asamblea General para poder determinar si se cumplen los requisitos (de amplia participación y mayoría a favor así como su contenido referido al Derecho internacional más allá de los principios de su Tratado constitutivo) para considerarla prueba de una práctica Estatal en la materia ${ }^{70}$.

\section{A MOdo de CONCluSión: "El FUTURo" de NACIONES Unidas y LAS Catástrofes}

A modo de cierre del presente trabajo exponemos algunos planteamientos referidos a las fortalezas y las debilidades del papel jugado por Naciones Unidas en materia de catástrofe y que ha sido expuesto en sus ejes principales en las páginas que preceden.

Los logros alcanzados por la Organización en las últimas décadas son evidentes. Se ha puesto el foco en la adopción de normas, principios y reglas de conducta aplicables a situaciones de catástrofe natural, antrópica o mixta. Además, se han establecido

\footnotetext{
${ }^{69}$ GonZÁlez Campos, J.-D., SÁnchez RodrígueZ, L.-I. y AndrÉs SÁEnZ De SANTA MaRÍa, P., Curso de Derecho Internacional Público, $4^{\mathrm{a}}$ edición, Thomson-Civitas, Navarra, 2008, p. 316; en el mismo sentido, AndRÉs SÁenz de Santa María, P., Sistema de Derecho Internacional Público, $4^{\mathrm{a}}$ ed., Thomson ReutersCivitas, Navarra, 2016, pp. 207-209.

${ }^{70}$ Respecto de los Principios rectores de la prestación de asistencia a los que se refieren las Resoluciones de la Asamblea General 43/131, 45/100 y 46/182, se puede afirmar este valor jurídico vinculante pretendido dada su formulación y el consenso en su aprobación; en este sentido secundamos el planteamiento expuesto por TORROJA MATEU, H., «Estrategia...» op. cit., pp. 253-257, especialmente en 253 y 254.
} 
mecanismos para la coordinación de las acciones en respuesta a catástrofes, situándose Naciones Unidas como el principal coordinador de la asistencia a tales emergencias.

Sin embargo, siguen pensando las deficiencias en este sector. La ausencia de un marco normativo general, perspicuo y vinculante sobre catástrofes, demuestra la dualidad seguida por los Estados en el seno de la Asamblea General y la poca voluntad de la sociedad internacional de dotar de regulación jurídica a las catástrofes. Recordemos que hasta la fecha sólo existen dos Tratados internacionales multilaterales de ámbito universal en la materia: el Convenio de Tampere sobre el suministro de recursos de telecomunicaciones para la mitigación de catástrofes y las operaciones de socorro en caso de catástrofe, de 18 de junio de 1998, y el Convenio Marco de Asistencia en Materia de Protección Civil, de 22 de mayo de 2000. Además, la falta de fondos económicos tanto ordinarios como extraordinarios destinados a la gestión de las catástrofes se sitúa como un mal endémico en estos asuntos. La labor de las Naciones Unidas, al igual que cualquier otra Organización, queda limitada por los recursos financieros de que la doten sus miembros

En este contexto, resulta indudable la necesidad de establecer unos estándares internacionales de regulación aplicables a la sociedad internacional, y que los mismos sean elaborados teniendo en cuenta la experiencia y necesidades a nivel nacional, regional y local; pues es en estos niveles dónde puede atajarse de mejor manera los conocimientos y la experiencia sobre riesgos así como las vulnerabilidades, de modo que se superen las diferencias en materia de desarrollo de las distintas regiones afectadas por la catástrofe.

En conclusión, el papel de Naciones Unidas ha quedado relegado a la coordinación internacional de la respuesta a las catástrofes a través de la UNOCHA que se convierte junto con el IASC en el nivel superior de coordinación en la respuesta a catástrofes. De este modo se pretende una mayor coherencia y eficiencia en las acciones llevadas a cabo por los distintos actores prestadores de asistencia (Estados, Organizaciones, ONG...). Sin embargo, la multitud de entes dentro de Naciones Unidas y sobre todo, fuera de su organigrama que pueden y prestan asistencia de uno u otro tipo requiere de un mayor esfuerzo de clarificación de funciones y de centralización de la 
asistencia de manera que ésta se proporcione de manera ágil, rápida y eficiente. Junto con el rol de coordinador de la Oficina, cobra cada vez mayor peso su función de «administración de donaciones» que debe solicitar y gestionar para la prestación de asistencia. No existe una atribución directa de fondos para la gestión internacional de catástrofes, (recuérdese que sólo se contemplan recursos extrapresupuestarios para las labores de coordinación de la asistencia), y ello es debido a que tampoco existe un órgano dedicado en exclusiva a tal gestión. Las distintas acciones posibles han sido asumidas por organismos especializados que actúan dentro de su ámbito (refugiados, infancia, desarrollo...), por lo que no existe ni una labor completa y general en este ámbito, ni per se una coordinación entre estas políticas y acciones. Para ello se requiere de organismos interinstitucionales como el IASC.

En nuestra opinión, la clave de bóveda se sitúa en definir con claridad qué función debe ejercer Naciones Unidas en materia de catástrofes. Si los Estados miembros entienden que su papel debe ser la centralización y coordinación a nivel internacional de la gestión de las catástrofes, entonces debe procederse a la creación de un ente con tales funciones que de forma general y comprensiva se dedique a la gestión integral en todas sus fases de estos fenómenos, no limitándose a la prevención y respuesta. Igualmente, se requiere que se establezcan recursos económicos, materiales y personales adecuados, suficientes y permanentes que permitan realizar su labor de manera eficiente y efectiva. Las catástrofes suponen un reto para la sociedad internacional del siglo XXI que afecta de manera directa a la seguridad humana. Es responsabilidad de los pueblos de las Naciones Unidas, tal y como reza el preámbulo de su Carta constitutiva, aunar sus esfuerzos para alcanzar mayores logros en materia de seguridad internacional, promover el progreso social y elevar el nivel de vida de la sociedad internacional.

\section{BIBLIOGRAFÍA}

ANDRÉS SÁENZ DE SANTA MARÍA, P., Sistema de Derecho Internacional Público, $4^{\mathrm{a}}$ ed., Thomson Reuters-Civitas, Navarra, 2016. 
BAUTISTA-HERNÁEZ, A., «Cooperación transfronteriza y cooperación internacional frente a catástrofes en las relaciones Luso-Españolas. Consideraciones en torno al Protocolo de Évora de 1992», en FERNÁNDEZ SÁNCHEZ, P.-A. y AZEREDO LOPES, J.-A., Seguridad medioambiental y cooperación transfronteriza. IV Encuentro LusoEspañol de Profesores de Derecho Internacional Público y Relaciones Internacionales, Atelier, Barcelona, 2015, pp. 49-82.

BAUTISTA-HERNÁEZ, A., «Recientes avances en la regulación de la Unión Europea en materia de catástrofes: el Mecanismo de Protección Civil de la Unión», en GARCÍA SAN JOSÉ, D., SÁNCHEZ PATRÓN, J.-M. y TORRES CAZORLA, M.-I. (Coords.), Bioderecho, Seguridad y Medioambiente. Biolaw, Security and Environment, Tirant lo Blanch, Valencia, 2015, pp. 173-194.

BAUTISTA-HERNÁEZ, A., «Reflexiones en torno a la Regulación Jurídica de la Reducción del Riesgo de Desastre (Disaster Risk Reduction) y la Práctica Internacional al respecto seguida por la UNESCO», en Ius et Scientia, Vol. 2, núm. 1 (2016), pp. 89107.

CARON, D.-D., et al. (ed.), The International Law of Disaster Relief, Cambridge University Press, Cambridge, 2014.

FERNÁNDEZ LIESA, C.-R. y OLIVA MARTÍNEZ, J.-D., El Derecho Internacional y la cooperación frente a los desastres en materia de Protección Civil, Dirección General de Protección Civil y Emergencias, Madrid, 2012.

FISCHER, H., «International disaster response law treaties: trends, patters and lacunae», en FEDERACIÓN INTERNACIONAL DE SOCIEDADES DE LA CRUZ ROJA Y DE LA MEDIA LUNA ROJA, International Disaster Response Laws, Principles and Practice: Reflections, Prospects and Challenges, Ginebra, 2003. 
GONZÁLEZ CAMPOS, J.-D., SÁNCHEZ RODRÍGUEZ, L.-I. y ANDRÉS SÁENZ DE SANTA MARÍA, P., Curso de Derecho Internacional Público, $4^{\mathrm{a}}$ edición, ThomsonCivitas, Navarra, 2008.

GUTTRY, A. DE, GESTRI, M., VENTURINI, G. (Eds.), International Disaster Response Law, TMC Asser Press, The Hague (The Netherlands), 2012.

HOFFMAN, M.-H., «What is the Scope of International Disasters Response Law?», en FEDERACIÓN INTERNACIONAL DE SOCIEDADES DE LA CRUZ ROJA Y DE LA MEDIA LUNA ROJA, International Disaster Response Laws, Principles and Practice: Reflections, Prospects and Challenges, Ginebra, 2003.

LIENHARD, C., «Pour un droit des catatrophes», Recueil Dalloz, núm. 13, 30 de marzo de 1995, recurso electrónico, n.p. (la versión impresa comprende pp. 91-102).

TORROJA MATEU, H. «La Organización de las Naciones Unidas ante los desastres naturales», en REQUENA HIDALGO, J. y CAMPINS ERITJA, M. (Coord.), De las catástrofes ambientales a la cotidianidad urbana: la gestión de la seguridad y el riesgo. II Coloquio Hispano-Canadiense de Barcelona, Universidad de Barcelona, Barcelona, 2000.

TORROJA MATEU, H., «Estrategia Internacional para la seguridad humana en los desastres naturales», en Araucaria. Revista Iberoamericana de Filosofía, Política y Humanidades, núm. 36 (2016).

THOUVENIN, J.-M., «L'internationalisation des secours en cas de catastrophe naturelle», en Revue General de Droit International Publique, Tomo 102, vol. 2 (1998), pp. $340-341$. 